\title{
Nanospherical heterolayer in strong electrostatic field
}

\author{
V. A. Harutyunyan
}

Received: 28 October 2011/Accepted: 6 March 2012/Published online: 30 March 2012

(c) The Author(s) 2012. This article is published with open access at Springerlink.com

\begin{abstract}
The influence of a strong homogeneous electric field on the states of charge carriers in the structure of quantum dot-quantum well (QDQW) is studied theoretically. It is shown that a strong external field changes radically the character of carrier motion in the structure and leads to an additional field-localization of the particle along the polar angle variable. An explicit form of the wave functions and energy spectrum of single-particle states in the structure in the presence of an external field is obtained. The possibilities of experimental and operational applications of the theoretical results obtained for the study of core/layer/shell structures as well as of hollow spheres are also shown.
\end{abstract}

Keywords Nanoheterolayer - Electrostatic field . Localization

\section{Introduction}

The rapid progress in the techniques of semiconductor material growth makes it possible to fabricate various quantum nanostructures. Research on semiconductor nanoparticles has become a wide and interdisciplinary field of science over the last two decades. Low-dimensional quantized semiconductor nanostructures from quantum well $(\mathrm{QW})$, through quantum wire $(\mathrm{QWr})$ to quantum dot $(\mathrm{QD})$, exhibited unique physical properties differing substantially from those of the bulk solids. These systems hold promise for opto- and nanoelectronics, and are extensively

V. A. Harutyunyan $(\bowtie)$

State Engineering University of Armenia, Gyumri Branch,

2 M.Mkrtchyan St., 3103 Gyumri, Armenia

e-mail: volharut@mail.ru investigated due to their remarkable applications (electronic devices, tunable optoelectronic elements, lasers, telecommunication systems, etc. (Balandin and Wang 2006; Schmid 2010; Rogach 2008; Hofman 2009)). Recently, a wide variety of new and promising heteroquantum dots (inhomogeneous QDs) named quantum dot-quantum wells (QDQWs) have been fabricated and studied both theoretically and experimentally. They mainly consisted of II-VI, IV-VI, and III-V compounds and can be presented, such as $\mathrm{CdS} / \mathrm{HgS} / \mathrm{CdS}, \mathrm{ZnS} / \mathrm{CdS} / \mathrm{ZnS}, \mathrm{CdSe} / \mathrm{HgSe} / \mathrm{CdSe}, \mathrm{CdTe} /$ $\mathrm{HgTe} / \mathrm{CdTe}, \mathrm{CdS} / \mathrm{PbS} / \mathrm{CdS}, \mathrm{PbSe} / \mathrm{CdS} / \mathrm{PbSe}, \mathrm{PbSe} / \mathrm{CdSe} /$ $\mathrm{PbSe}, \mathrm{AlAs} / \mathrm{GaAs} / \mathrm{AlAs} /, \mathrm{InP} / \mathrm{InAs} / \mathrm{InP}$, etc. heterostructures (see for e.g., (Balandin and Wang 2006; Schmid 2010; Rogach 2008; Hofman 2009; Eychmuller et al. 1993; Schooss et al. 1994; Mews et al. 1996; Hause et al. 1993; Bryant 1995; Little et al. 1998; Braun et al. 2001; Bryant and Jaskolski 2003; Perez-Conde and Bhattacharjee 2006; Berezowsky et al. 2006; Weng et al. 2009; Guo-Yi 2004; Royo et al. 2007; Brovelli et al. 2011; Jia 2011; SalmanOgli and Roatami 2011) and references therein).The QDQW structure involves an onion-like nanosystem composed of a quantum dot core surrounded by two or more shells of alternating narrower and wider band gap materials. The original feature of QDQW is that their physical properties can be controlled and can be tuned by changing the core radius, the thickness of the quantized layer and the size of the outermost shell. These types of hierarchical nanostructures introduce a new dimension to band gap engineering (modification of band gap profile, charge-carrier properties, and luminescence feature). In the case of QDQW structures additional tuning of the energy levels and carrier wave functions can be achieved by varying the composition and the width of the QW surrounding the QD. Quantum dotquantum well-type heterophase nanostructures have also attracted much attention due to their interesting physics and

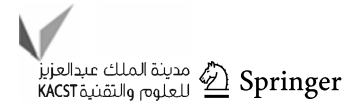


possible electronic and optical applications. They also are promising candidates for use in optoelectronic elements and, in particular, can be used in a variety of applications involving optical devices, such as photodetectors or photovoltaic cells (Kim et al. 2006; Cat et al. 2009; Prasad 2004). A QDQW structure can be formed by coating QD, preferably spherical shaped (core), with a thin layer of a different semiconductor that has a smaller energy gap and then further covered by a thick layer (shell) of the same semiconductor as the dot, which has a large energy gap. For e.g., in the CdS/ $\mathrm{HgS} / \mathrm{CdS}$ QDQW structure the core of CdS (band gap of $2.5 \mathrm{eV}$ ) is surrounded and covered with a quantized layer of the narrower band gap material $(\mathrm{HgS}$, band gap of $0.5 \mathrm{eV}$ for $\beta-\mathrm{HgS}$ ), which is then covered by another thick CdS layer (shell). In this composition the $\beta-\mathrm{HgS}$ layer provides a quantum well for both electrons and holes, whereas $\mathrm{CdS}$ core- and shell-components of the system play a role of barriers (Eychmuller et al. 1993; Schooss et al. 1994; Mews et al. 1996; Hause et al. 1993; Bryant 1995; Little et al. 1998; Braun et al. 2001), i.e., along the "global" confinement of the entire nanoparticle, the additional "local" confinement in the internal quantum well of the sample takes place in QDQW structures. Several experimental and theoretical investigations of this system have shown that after optical excitation the electrons and holes in this heterostructure are localized in the HgS QW (Guo-Yi 2004; Royo et al. 2007; Braun et al. 2002). Other well-known and widely studied QDQW systems, for e.g., ZnS/CdS/ZnS (Bryant and Jaskolski 2003; Perez-Conde and Bhattacharjee 2003, 2006; Cao et al. (2005), CdTe/HgTe/CdTe (Kim et al. 2006; Cat et al. 2009; Prasad 2004)), also exhibit similar properties. So, it is clear that the study of states of charge carriers in the internal QW of such barrier/well/barrier structures under the different physical conditions is of a separate interest. From this point of view in several papers in the frameworks of various approximations are being discussed the electronic, hole (Eychmuller et al. 1993; Schooss et al. 1994; Mews et al. 1996; Hause et al. 1993; Bryant 1995; Little et al. 1998; Braun et al. 2001; Bryant and Jaskolski 2003; Perez-Conde and Bhattacharjee 2006), excitonic (Kim et al. 2006; Cat et al. 2009; Prasad 2004; Heng and Jun-Jie 2004; Kai and Xia (1998); Jun-Jie (2002)) and phonon (Berezowsky et al. 2006; Weng et al. 2009; Guo-Yi 2004; Royo et al. 2007) states, as well as the optical absorption and luminescence spectra (Eychmuller et al. 1993; Schooss et al. 1994; Mews et al. 1996; Hause et al. 1993; Bryant 1995; Little et al. 1998; Braun et al. 2001; Kim et al. 2006; Cat et al. 2009; Prasad 2004; Porteanu et al. 2001; Fang et al. 2010) of QDQW structures.

At the same time, it is well recognized that the electronic, kinetic, optical, and number of other properties of nanostructures (as well as those of bulk samples) are strongly affected by external static fields. We focus on the external uniform electric field. In this aspect, there are a relatively small number of publications that focus on the study of modulating influence of external uniform electric field on the QDQW structures. In particular, in the framework of the available scientific literature, we could not find references concerning the electro-absorption in QDQWs.

As for theoretical works, the magnetic and electric field effects on the binding energy of a shallow donor confined to move in spherical QDQW is studied in Rahmani et al. (2009), and quadratic electro-optic effects and electroabsorption process in multilayer nanoshells is discussed in Bahari and Moghadam (2011). In the previous works of the author with co-authors the influence of weak external uniform (Harutyunyan 2003, 2007; Harutyunyan et al. 2004a) and radial (Harutyunyan 2002; Harutyunyan et al. 2004b) electrostatic fields on the electronic and optical properties of QDQWs are examined theoretically. In the present paper, we studied theoretically the influence of strong external homogeneous electrostatic field on the single-electron states in spherical QDQW and, correspondingly, on the interband optical absorption spectra of the system.

\section{Modeling approach and approximations}

We assume that for the considered core/layer/shell QDQW structure the following conditions are satisfied:

1. both the core and the shell are made from the same material,

2. the material of layer has narrower band-gap than the material of the core (shell),

3. for overlapping band gaps of contacting materials the interface band offset, counted from the vacuum level, is much greater than the charge carriers' confinement energy of radial motion in the layer.

In this sense a typical heterostructure is, for example, a direct-band $\mathrm{CdS} / \mathrm{HgS} / \mathrm{CdS}$ system.

In this case, from the quantum mechanical point of view the QDQW can be presented as a structure of barrier/well/ barrier. Correspondingly, the confinement potential of quantized heterolayer of the structure can be approximated, in radial direction, by the quantum well "rolled-up into a sphere" (Harutyunyan 2002, 2003, 2007; Harutyunyan et al. 2004a, b):

$U_{\text {conf }}(r)=\left\{\begin{array}{ccc}0, & \text { when } \quad R_{1}<r<R_{2} \\ \infty, & \text { when } \quad r \leq R_{1}, r \geq R_{2}\end{array}\right.$

Here $R_{1}$ and $R_{2}$ are the inner and outer radii of the layer, respectively. Note that a model approach (1) can be applied with great accuracy to the case of hollow nanospheres $\left(\mathrm{SnO}_{2}, \mathrm{ZnO}\right.$, InP etc.) also (Fan et al. 2006; Zhang et al. 2008; Zhu and Qian 2010; Wang et al. 2011). 
It is also assumed, that the layer is "thin enough" and lies fairly far from the centre of the system:

$L \ll R_{1}, R_{2} ; \quad L=R_{2}-R_{1}$

At the same time we will also restrict the analysis with the strong confinement (strong quantization) regime when the Coulomb interaction between the electron and hole in the layer can be neglected in comparison with the confinement energies of charge carriers. This means that the thickness of the layer $L$ is sufficiently smaller than the Bohr radius $a_{L}$ of the bulk exciton in the material of the layer: $L^{2} \ll a_{L}^{2}$.

We will carry out theoretical analysis within isotropic effective-mass approximation in the framework of simple parabolic non-degenerate direct-band model. Let us assume that the external uniform field $\vec{F}=\vec{F}(0,0, F)$ is directed along the $z$-axis. In general, if the static dielectric constants of the core $\left(\varepsilon_{1}\right)$, layer $\left(\varepsilon_{2}\right)$ and shell $\left(\varepsilon_{3}\right)$ are different, then in spherical coordinates $(r, \vartheta, \varphi)$ we obtain the following expression for electrostatic energy of particle in the layer (Smythe 1968):

$V(r, \vartheta)=q F\left(B r+\frac{C}{r^{2}}\right) \cos \vartheta \equiv V(r) \cos \vartheta$

Here $q$ is the charge of particle, and the constants $B$ and $C$, that are specified from the boundary conditions for electrostatic potential and its derivatives at $r=R_{1}$ and $r=R_{2}$, are as follows:

$$
\begin{aligned}
B= & \frac{C}{R_{1}^{3}} \frac{\varepsilon_{3}+2 \varepsilon_{2}}{\varepsilon_{2}-\varepsilon_{1}} ; \\
C & =\frac{3 \varepsilon_{1}\left(\varepsilon_{2}-\varepsilon_{1}\right) R_{1}^{3} R_{2}^{3}}{\left(\varepsilon_{2}+2 \varepsilon_{1}\right)\left(\varepsilon_{3}+2 \varepsilon_{2}\right) R_{2}^{3}+2\left(\varepsilon_{1}-\varepsilon_{2}\right)\left(\varepsilon_{2}-\varepsilon_{3}\right) R_{1}^{3}} .
\end{aligned}
$$

The corresponding Schrodinger equation for a particle in the layer can be written as

$$
\begin{aligned}
- & \frac{\hbar^{2}}{2 \mu} \Delta(r, \vartheta, \varphi) \Psi(r, \vartheta, \varphi)+V(r) \cos \vartheta \Psi(r, \vartheta, \varphi) \\
& =E \Psi(r, \vartheta, \varphi)
\end{aligned}
$$

with boundary conditions

$\Psi\left(r=R_{1}\right)=\Psi\left(r=R_{2}\right)=0$

In Eq. (6) $\Delta(r, \vartheta, \varphi)$ is Laplace operator in spherical coordinates, and $\mu$ and $E$ are the effective mass and full energy of the particle in the layer, respectively.

The solutions of Eq. (6) are sought in the form as

$$
\begin{aligned}
\Psi(r, \vartheta, \varphi) & =U(r, \vartheta) f(\varphi) \\
& \equiv U(r, \vartheta) \frac{e^{i|m| \varphi}}{\sqrt{2 \pi}} ;(m=0, \pm 1, \pm 2, \ldots)
\end{aligned}
$$

We obtain now instead of Eq. (6):

$$
\begin{aligned}
- & \frac{\hbar^{2}}{2 \mu}\left[\frac{1}{r^{2}} \frac{\partial}{\partial r}\left(r^{2} \frac{\partial}{\partial r}\right)-\frac{1}{r^{2} \sin \vartheta}\left(\sin \vartheta \frac{\vartheta}{\partial \vartheta}\right)-\frac{m^{2}}{r^{2} \sin \vartheta}\right] \\
& \times U(r, \vartheta)+V(r) \cos \vartheta=E U(r, \vartheta) .
\end{aligned}
$$

Taking into account the condition (2) for the layer, the functions $V(r)$ and $r^{-2}$ in Eq. (9) can be considered as slowly varying in interval $\left[R_{1}, R_{2}\right]$. This makes it possible to solve the Eq. (9) in the adiabatic approximation. Separating in Eq. (9) the fast (radial) part and introducing simultaneously $U(r, \vartheta)$ and $E$ in the forms

$U(r, \vartheta)=\Phi(r) \chi(\vartheta) ; E=E_{\mathrm{rad}}+E_{\mathrm{ang}}$

we obtain for the wave function $\Phi(r)$ and energy $E_{\text {rad }}$ of radial motion of particle in the layer (Harutyunyan 2002, 2003, 2007; Harutyunyan et al. 2004a; b):

$$
\begin{aligned}
\Phi_{n}(r) & \cong \sqrt{\frac{2}{L}} \frac{\sin \frac{\pi n}{L}\left(r-R_{1}\right)}{r} ; E_{r a d} \equiv E_{n} \\
& \cong \frac{\pi^{2} \hbar^{2} n^{2}}{2 \mu L^{2}} ;(n=1,2,3, \ldots)
\end{aligned}
$$

Accordingly, for the angular wave function $\chi(\vartheta)$ we obtain the equation

$$
\begin{aligned}
- & \frac{\hbar^{2}}{2 \mu}\left[\frac{1}{R_{n}^{2}} \frac{1}{r^{2} \sin \vartheta}\left(\sin \vartheta \frac{\vartheta}{\partial \vartheta}\right) \frac{m^{2}}{R_{n}^{2} \sin \vartheta}\right] \chi(\vartheta)+V_{0} \cos \vartheta \\
= & E_{\text {ang }} \chi(\vartheta),
\end{aligned}
$$

or

$$
\begin{array}{r}
\frac{1}{\sin \eta} \frac{\partial}{\partial \eta}\left(\sin \eta \frac{\partial}{\partial \eta}\right) \chi(\eta)-\frac{m^{2}}{\sin ^{2} \eta} \chi(\eta) \\
+\frac{2 \mu R_{n}^{2}}{\hbar^{2}}\left(E_{\text {ang }}+V_{0} \cos \eta\right) \chi(\eta)=0
\end{array}
$$

Here $\eta=\pi-\vartheta$,

$$
\begin{aligned}
& R_{n}^{-2} \equiv\left\langle\frac{1}{r^{2}}\right\rangle \cong R_{1}^{-2}\left[1-\frac{L}{R_{1}}+\frac{L^{2}}{R_{1}^{2}}\left(1-\frac{3}{2 \pi^{2} n^{2}}\right)\right] \\
& V_{0} \equiv\langle V(r)\rangle=\left\langle B r+\frac{C}{r^{2}}\right\rangle \cong B R_{1}\left(1+\frac{L}{2 R_{1}}\right)+\frac{C}{L^{2}} \ln \frac{R_{2}}{R_{1}}
\end{aligned}
$$

are the results of adiabatic averaging of "slow" functions $r^{-2}$ and $V(r)$ over the states of "fast" motion (Exp. (11)).

\section{Single-particle states in the layer in the presence of strong external field}

Let us assume that in Eqs. (12), (13) the following condition takes place 
$\frac{V_{0}}{E_{\text {ang }}} \gg 1$.

Physically this condition means that the depth of potential well $V(r, \vartheta) \cong V_{0} \cos \vartheta=-V_{0} \cos \eta$, which creates the external field along the angular variable, is much larger than the energy of the particle along the corresponding direction. Condition (16) allows us to present a potential well for the lower energy levels in the form

$V_{0} \cos \eta \approx V_{0}\left(1-\eta^{2} / 2\right)$

Correspondingly, taking into account the possibility of expansion of Exp. (17), instead the Eq. (13) we obtain the following new equation:

$$
\begin{gathered}
\eta^{2} \frac{\mathrm{d}^{2} \chi}{\mathrm{d} \eta^{2}}+\eta \frac{\mathrm{d} \chi}{\mathrm{d} \eta}+\alpha \eta^{4} \chi+\beta \eta^{2} \chi-m^{2} \chi=0 ; \\
{\left[\alpha=-\frac{2 \mu R_{n}^{2} V_{0}}{\hbar^{2}}, \beta=\frac{2 \mu R_{n}^{2}\left(E_{\mathrm{ang}}+V_{0}\right)}{\hbar^{2}}\right] ;}
\end{gathered}
$$

This equation can be reduced to an equation of hypergeometric type (Zaytsev and Polyanin 2001), and its solution can be presented in the following form:

$$
\begin{aligned}
\chi_{k, m}(\eta) & C_{k,|m|} \eta^{|m|} \exp \left(-\frac{\sqrt{-\alpha}}{2} \eta^{2}\right){ }_{1} F_{1}\left(-k,|m|+1, \sqrt{-\alpha} \eta^{2}\right) \\
& \int_{0}^{\pi}\left|\chi_{k, m}(\eta)\right|^{2} \sin \eta d \eta=1 ; \quad(k=0,1,2, \ldots)
\end{aligned}
$$

Here $C_{k,|m|}$ is a normalized constant, and ${ }_{1} F_{1}(a, b, x)$ is Kummer confluent hipergeometric function. Taking into account the Exps. (16), (17) for $C_{k,|m|}$ we obtain (Landau and Lifshitz 1977):

$$
\begin{aligned}
C_{k,|m|} & =\sqrt{\frac{2}{J_{k,|m|}}}\left(\frac{\hbar^{2}}{2 \mu R_{n}^{2} V_{0}}\right)^{\frac{|m|+1}{2}} ; J_{k,|m|} \\
& =\int_{0}^{\infty} x^{|m|} e^{-x}\left|{ }_{1} F_{1}(-k,|m|+1, x)\right|^{2} d x \\
& =\frac{|m| ! k !}{(|m|+1)(|m|+2) \ldots(|m|+k)}
\end{aligned}
$$

Correspondingly, the particle's energy along the polar angle $(\vartheta)$ variable will be presented in the following form:

$$
\begin{array}{r}
E_{\text {ang }} \equiv E_{k,|m|}=\sqrt{\frac{\hbar^{2} V_{0}}{2 \mu R_{n}^{2}}}(2 k+|m|+1) ; \\
(k=0,1,2, \ldots ; m=0, \pm 1, \pm 2, \ldots)
\end{array}
$$

Here and below we omit the additive energy term $V_{0}$, since it only leads to a shift of the origin of energy and, consequently, has no real physical meaning.

\section{Discussion of results}

Let us now discuss the results obtained in the work. In the absence of a field with other similar conditions, the state of the carriers in the layer are described by the following wave functions (Harutyunyan 2002, 2003, 2007; Harutyunyan et al. 2004a, b)

$$
\begin{aligned}
\Psi_{n, l, m}^{(0)}(r, \vartheta, \varphi) & \cong \sqrt{\frac{2}{L}} \frac{\sin \frac{\pi n\left(r-R_{1}\right)}{L}}{r} Y_{l, m}(\vartheta, \varphi) \\
& \equiv \Phi_{n}^{(0)}(r) Y_{l, m}(\vartheta, \varphi)
\end{aligned}
$$

where $n=1,2, \ldots$ is the radial quantum number, $Y_{l, m}(\vartheta, \varphi)$ are normalized spherical functions (Landau and Lifshitz 1977) and $l, m$ are orbital and azimuthal quantum numbers, respectively. The energy spectrum of charge carriers in the absence of field is at the same time as the substructure

$E_{n, l}^{(0)} \cong \frac{\pi^{2} \hbar^{2} n^{2}}{2 \mu L^{2}}+\frac{\hbar^{2} l(l+1)}{2 \mu R_{n}^{2}} ;$

In this substructure each $n$th level with a given $l$ has $2 l+1$-fold degeneracy by the azimuthal quantum number $m$, which is not explicitly included in the expression of energy.

From the form of the Exps. (19), (20) it is clear that a strong external electrostatic field creates in a layer completely new physical situation. As we see, if the energy obtained by a charge carrier from an external field, is much more than the energy of the orbital motion in the layer, the motion character and, correspondingly, the energy spectrum of a particle along the angular variables $\vartheta$ and $\varphi$ are changed. A strong external field creates a new deep potential well along the polar variable $\vartheta$ As a consequence:

1. first, along with the quantum confinement in the radial direction, the charge carriers in the layer are additionally localized also with respect to their polar-angular motion. The particle under action of a strong external field, vibrates in a narrow angular cone of the polar variable $\vartheta$. This case is physically similar to the case when a rotation of an eccentric wheel is changed to a vibration with the gravity force increasing.

2. Rotational motion by the azimuthal angle $\varphi$ in the presence of an external field is preserved, but this rotation does not occur over the entire surface of the sphere. The rotation occurs now around the circumference of the polar angle cone base.

3. At the same time the strong external field separates electron and hole spatially along the field's direction and traps them to the opposite ends of the spherical layer's diameter. The localization cones of opposite charges are disposed at opposite edges of the layer diameter directed along the external field. 
4. The energy of the angular motion of charge carriers is now determined by the explicit dependence on the azimuthal number $m=0, \pm 1, \pm 2, \ldots$ and on a new quantum number $k=0,1,2, \ldots$ which now characterizes the particle's motion along the polar angle.

For the complete wave function and for the full energy of the particle in the layer in the presence of external field can be written now:

$\Psi(r, \vartheta, \varphi)=\sqrt{\frac{2}{L}} \frac{\sin \frac{\pi n\left(r-R_{1}\right)}{L}}{r} \chi(\vartheta) \frac{e^{i|m| \varphi}}{\sqrt{2 \pi}}$
$E \equiv E_{\mathrm{rad}}+E_{\mathrm{ang}}=\frac{\pi^{2} \hbar^{2} n^{2}}{2 \mu L^{2}}+\sqrt{\frac{\hbar^{2} V_{0}}{2 \mu R_{n}^{2}}}(2 k+|m|+1)$

As we can see, the energy substructure of a particle in the layer in the presence of a strong external field is characterized by a set of three quantum numbers. Note also, that the presence of an external field leads to an explicit dependence of the particle wave functions on the values of the effective masses of charge carriers.

\section{Conclusions}

On the results obtained in this study we can conclude the following:

- the suggested theoretical model is physically adequate to the system under consideration and allows to get results in analytical form,

- the energy spectrum of charge carriers in QDQW in the presence of external strong homogeneous electric field is strongly discrete; consequently the electro-optical absorption in the system will be strictly resonant,

- under the influence of strong external field the rotational motion of carriers around the circumference of the sphere disappears; instead the electron and hole begin to oscillate in a narrow angular cone at the opposite ends of a diameter in the direction of the field, at the same time making a rotation around the circumference of the base of the cone,

- the external field leads to a spatial separation of opposite charge carriers, which reduces the probability of radiative recombination of electron-hole pair.

- If the material and geometrical sizes of QDQW are known, the results of above theoretical calculations could be used to carry out appropriate experiments;

- it is possible to modify the optical-energy parameters of the system by variation of geometrical sizes of the sample and the intensity of external field in a controlled manner; at the same time the dependence of absorption parameters upon effective masses of carriers opens a possibility to define its values experimentally in the presence of external electric field.

As a consequence, we can conclude that in the application plan there is a real opportunity to use the theoretical results obtained for the geometrical and electrical tuning of optical and other parameters of QDQW.

Open Access This article is distributed under the terms of the Creative Commons Attribution License which permits any use, distribution, and reproduction in any medium, provided the original author(s) and the source are credited.

\section{References}

Bahari A, Moghadam FR (2011) Quadratic electro-optic effects and electro-absorption process in multilayer nanoshells. Phys Scr 84:019802

Balandin AA, Wang KL (eds) (2000-2006) Handbook of semiconductor nanostructures and nanodevices (5-volume set). American Scientific, Valencia, p 2500

Berezowsky J, Gywat O, Meier F, Battaglia D, Peng X, Awschalom DD (2006) Initialization and read-out of spins in couplet coreshell quantum dot. Nat Phys 2:831

Braun M, Burda C, El-Sayed M (2001) Variation of the thickness and number of wells in the $\mathrm{CdS} / \mathrm{HgS} / \mathrm{CdS}$ quantum dot quantum well system. J Phys Chem A105:5548

Braun M, Link S, Burda C, El-Sayed M (2002) Determination of the localization times of electron and holes in the $\mathrm{HgS}$ well in a CdS/ $\mathrm{HgS} / \mathrm{CdS}$ quantum dot-quantum well nanoparticle. Phys Rev B66:205312

Brovelli S, Schaller RD, Crooker SA, Garcia-Santamaria F, Chen Y, Vishvanatha R, Hollingsworth JA, Htoon H, Klimov VI (2011) Nano-engineering electron-hole exchange interaction controls exciton dinamics in core-shell semiconductor nanocrystals. Nat Commun. doi:10.1038/ncomms1281

Bryant GW (1995) Theory for quantum-dot quantum wells: pair correlation and internal quantum confinement in nanoheterostructure. Phys Rev B52:R16997

Bryant GW, Jaskolski W (2003) Charge separation effects on the rate of nonradiative relaxation processes in quantum dots-quantum well heterostructures. Phys Rev B67:205320

Cao L, Huang Sh, Lu Sh, Lin J (2005) Effect of layer thickness on the luminescence properties of $\mathrm{ZnS} / \mathrm{CdS} / \mathrm{ZnS}$ quantum dot quantum well. J Col Interf Sci 284:516

Cat DT, Pucci A, Wandlet K (2009) Physics and engineering of new materials. Springer, Berlin, p 387

Eychmuller A, Mews A, Weller H (1993) A quantum-dot quantum well: CdS/HgS/CdS. Chem Phys Lett 208:59

Fan Ch, Song X, Yin Zh, Yu H, Sun S (2006) J Mater Sci 41:5696

Fang Y, Xiao M, Yao D (2010) Quantum size dependent optical nutation in $\mathrm{CdSe} / \mathrm{ZnS} / \mathrm{CdSe}$ quantum dot quantum well. Physica E 42:2178

Guo-Yi G (2004) Phonons in quantum-dot quantum well. Commun Theor Phys 42:609

Harutyunyan VA (2002) Effect of radial electric field on absorption in quantized spherical layer. Semiconductors 36:379

Harutyunyan VA (2003) Confined Stark effect and intraband transitions in semiconductor spherical layer. Phys Solid State 45:1342

Harutyunyan VA (2007) Optical transitions in semiconductor nanospherical layer under thepresence of perturbating electrical field. Physica E 39:37 
Harutyunyan VA, Aramyan KS, Petrosyan HSh (2004a) Confined Stark effect and electroabsorption in semiconductor spherical layer. Semiconductors 38:335

Harutyunyan VA, Aramyan KS, Petrosyan HSh, Demirjan GH (2004b) Optical transitions in spherical quantized layer under the presence of radial electrical field. Physica E 24:173

Hause JW, Zhou HS, Honma I, Komiyama H (1993) Quantum confinement in semiconductor heterostructure nanometer-size particles. Phys Rev B 47:1359

Heng Zh, Jun-Jie Sh (2004) Cines Phys 13:2136

Hofman H (2009) Advanced nanomaterials course support. Powder Technology Laboratory IMX EPFL, Switzerland, p 270

Jia G (2011) Excitons properties and quantum qonfinement in CdS/ $\mathrm{ZnS}$ core/shell quantum dots. Optoel Adv Mater 5:738

Jun-Jie Sh (2002) Excitons in quantum-dot quantum-well nanoparticles. Ciines Phys 11:1286

Kai Ch, Xia J-B (1998) Spatially separated excitons in quantum-dot quantum well structures. Phys Rev B57:9780

Kim D-W, Cho K, Kim H, Park B, Sung MY, Kim S (2006) Optoelectronic characteristics of $\mathrm{CdTe} / \mathrm{HgTe} / \mathrm{CdTe}$ quantum-dot quantum well nanoparticles. Solid State Commun 140:215

Landau LD, Lifshitz EM (1977) Quantum mechanics: non-relativistic theory. Course of theoretical physics. vol 3, 3rd edn. Pergamon, New York

Little RB, Burda C, Link S, Logunov S, El-Sayed MA (1998) Charge separation effects on the rate of nonradiative relaxation processes in quantum dots-quantum well heterostructures. J Phys Chem A102:6581

Mews A, Kadavanich AV, Banin U, Alivisatos AP (1996) Structural and spectroscopic investigations of $\mathrm{CdS} / \mathrm{HgS} / \mathrm{CdS}$ quantum dotquantum wells. Phys Rev B 53:R13242

Perez-Conde J, Bhattacharjee AK (2003) Electronic structure and optical properties of $\mathrm{ZnS} / \mathrm{CdS}$ nanoheterostructures. Phys Rev B67:235303

Perez-Conde J, Bhattacharjee AK (2006) Quantum dot quantum wells: a tight-binding study. Phys Status Solid A 203:1182
Porteanu HE, Lifshitz E, Pflughoeff M, Eychmuller A, Weller H (2001) Optical properties of $\mathrm{CdS} / \mathrm{HgS} / \mathrm{CdS}$ quantum dot-quantum well structures. Phys Status Solid B 226:219

Prasad PN (2004) Nanophotonics. Wiley, New Jersey, p 432

Rahmani K, Zorkani I, Condensed MJ (2009) Magnetic and electric field effects on the binding energy of a shallow donor in quantum dot-quantum well. Mater 11:35

Rogach AL (2008) Semiconductor nanocrystal quantum dots: synthesis, assemble, spectroscopy and applications. Springer, New York, p 372

Royo M, Planelles J, Pi M (2007) Effective mass and dielectric mismatch effects in spherical multishell quantum dots. Phys Rev B 75:033302

SalmanOgli A, Rostami A (2011) Nanopart J Res 13:1197

Schmid G (2010) Nanoparticles: from theory to application, 2nd edn. Wiley, Weinheim, p 533

Schooss D, Mews A, Eychmuller A, Weller H (1994) Quantum-dot quantum well $\mathrm{CdS} / \mathrm{HgS} / \mathrm{CdS}$ : theory and experiment. Phys Rev B 49:17072

Smythe WR (1968) Static and dynamic electricity, 2nd edn. McGrowHill, New York

Wang L, Yang H, Yu Shi R, Bao L, Liu B (2011) Synthesis, formation mechanism and electric property of hollow InP nanospheres. Appl Phys A 104:61

Weng I-Ch, Tsai Ch-L, Chr Y (2008) Specific heat of CdS/HgS/CdS quantum-dot quantum wells. J Appl Phys 103:064310

Zaytsev VF, Polyanin AD (2001) Obiknovennie differencialnie uravneniya (handbook). Fizmatlit, Moscow

Zhang Y, Shi E-W, Chen Zh-Zh, Xiao B (2008) Fabrication of ZnO hollow nanospheres and "jingle bell" shaped nanospheres. Mater Lett 62:1435

Zhu J, Qian X (2010) From 2-D CuO nanosheets to 3-D hollow nanospheres: interface-assisted synthesis, surface photovoltage properties and photocatalytic activity. J Solid State Chem 183:1632 